\title{
A CLASS OF SHAPE OPTIMIZATION PROBLEMS FOR SOME NONLOCAL OPERATORS
}

\author{
JULIÁN FERNÁNDEZ BONDER, ANTONELLA RITORTO AND ARIEL MARTIN SALORT
}

\begin{abstract}
In this work we study a family of shape optimization problem where the state equation is given in terms of a nonlocal operator. Examples of the problems considered are monotone combinations of fractional eigenvalues. Moreover, we also analyze the transition from nonlocal to local state equations.
\end{abstract}

\section{INTRODUCTION}

In this article, we consider shape optimization problems that in the most general form can be stated as follows: Given a cost functional $F$, and a class of admissible domains $\mathcal{A}$, solve the minimization problem

$$
\min _{A \in \mathcal{A}} F(A) \text {. }
$$

These types of problems have been extensively considered, and they arise in many fields and in many applications. The literature is very wide, from the classical cases of isoperimetrical problems to the most recent applications including elasticity and spectral optimization. Only to mention some references, we refer the reader to the books of Allaire [2], Bucur and Buttazzo [7], Henrot [19], Pironneau [27] and Sokołowski and Zolésio [34], where a huge amount of shape optimization problems are tackled.

In most of the existing references, the cost functional $F$ is given in terms of a function $u_{A}$ which is the solution of a state equation to be solved on $A$ of the form

$$
F(A)=\int_{A} j\left(\nabla u_{A}, u_{A}, x\right) d x
$$

Typically, this state equation is an elliptic PDE.

In recent years there has been an increasing amount of interest in nonlocal problems due to several interesting applications that include some physical models $[14,15,17,23,26,36]$, finance $[1,24,31]$, fluid dynamics [10], ecology [20, 25, 30] and image processing [18].

However, there are only a handful of results of shape optimization problems of the form (1.1) where the state equation involves a nonlocal operator instead of an elliptic PDE.

For instance, in [33], the authors extend the well-known Faber-Krahn inequality to the fractional case and as a simple corollary, they solve problem (1.1) in the case where $F(A)=\lambda_{1}^{s}(A)$ where $\lambda_{1}^{s}(A)$ is the first eigenvalue of the Dirichlet fractional

2010 Mathematics Subject Classification. 35R11, 49Q10.

Key words and phrases. Fractional partial differential equations, shape optimization. 
laplacian and the class $\mathcal{A}$ is the class of open sets of fixed measure. (See next section for precise definitions).

In [4] the authors consider again the class $\mathcal{A}$ of open sets of fixed measure and $F(A)=\lambda_{2}^{S}(A)$ and prove that problem (1.1) does not have a solution. In fact, a minimization sequence of domains consists of a sequence of balls of the same measure where the distance of the centers diverges.

Finally, in [16], the authors take the class $\mathcal{A}$ of measurable sets of fixed measure contained in a fixed open set $\Omega$ and the cost functional $F(A)=\lambda_{1}^{s}(\Omega \backslash A)$ where in this case, $\lambda_{1}^{s}(\Omega \backslash A)$ is the first eigenvalue of the fractional laplacian with Dirichlet condition on $A$ and Neumann condition in $\mathbb{R}^{n} \backslash \Omega$.

For other recent shape optimization problems where the state equation is nonlocal, see [8, 12, 21, 22, 29], and references therein.

The purpose of this article is to consider a family of minimization problems of the form (1.1) for costs funcions $F$ under some natural assumptions that includes the particular cases mentioned above. These natural assumptions are similar to those considered in [9] where the authors addressed this problem when the state equation is given in terms of an elliptic PDE. Roughly speaking, these assumptions are:

- monotonicity with respect to the inclusion and

- lower semicontinuity with respect to a suitable defined notion of convergence of domains.

Observe that the results of [4] put a restriction on the classes of admissible domains that one needs to consider if you want to obtain a positive result. This is mainly due to the fact that taking a domain with two connected components and making these components go far away from each other makes the nonlocal energy decrease. So, in the spirit of [9] we restrict ourselves to the class $\mathcal{A}$ of open sets of fixed measure that are contained in a fixed box $Q \subset \mathbb{R}^{n}$.

Under these conditions, we are able to recover the results of [9] in the fractional setting and, moreover, we analyze the transition from the fractional case to the classical elliptic PDE case proving convergence of the minima and of the optimal shapes.

\section{Setting of the Problem}

2.1. Some preliminaries and notation. Given $s \in(0,1)$ we consider the fractional laplacian, that for smooth functions $u\left(C^{2}\right.$ and bounded are enough, see [13]) is defined as

$$
\begin{aligned}
(-\Delta)^{s} u(x) & :=c(n, s) \text { p.v. } \int_{\mathbb{R}^{n}} \frac{u(x)-u(y)}{|x-y|^{n+2 s}} d y \\
& =-\frac{c(n, s)}{2} \int_{\mathbb{R}^{n}} \frac{u(x+z)-2 u(x)+u(x-z)}{|z|^{n+2 s}} d z
\end{aligned}
$$

where $c(n, s):=\left(\int_{\mathbb{R}^{n}} \frac{1-\cos \zeta_{1}}{|\zeta|^{n+2 s}} d \zeta\right)^{-1}$ is a normalization constant.

The constant $c(n, s)$ is chosen in such a way that the following identity holds,

$$
(-\Delta)^{s} u=\mathcal{F}^{-1}\left(|\xi|^{2 s} \mathcal{F}(u)\right)
$$


for $u$ in the Schwarz class of rapidly decreasing and infinitely differentiable functions, where $\mathcal{F}$ denotes the Fourier transform. See [13, Proposition 3.3].

The natural functional setting for this operator is the fractional Sobolev space $H^{s}\left(\mathbb{R}^{n}\right)$ defined as

$$
\begin{aligned}
H^{s}\left(\mathbb{R}^{n}\right) & :=\left\{u \in L^{2}\left(\mathbb{R}^{n}\right): \frac{u(x)-u(y)}{|x-y|^{\frac{n}{2}+s}} \in L^{2}\left(\mathbb{R}^{n} \times \mathbb{R}^{n}\right)\right\} \\
& =\left\{u \in L^{2}\left(\mathbb{R}^{n}\right): \int_{\mathbb{R}^{n}}\left(1+|\xi|^{2 s}\right)|\mathcal{F}(u)(\xi)|^{2} d \xi<\infty\right\}
\end{aligned}
$$

which is a Banach space endowed with the norm $\|u\|_{s}^{2}:=\|u\|_{2}^{2}+[u]_{s}^{2}$, where the term

$$
[u]_{s}^{2}:=\iint_{\mathbb{R}^{n} \times \mathbb{R}^{n}} \frac{|u(x)-u(y)|^{2}}{|x-y|^{n+2 s}} d x d y
$$

is the so-called Gagliardo semi-norm of $u$.

Due to the nonlocal nature of the operator $(-\Delta)^{s}$, when dealing with Dirichlettype problems in a open bounded set $\Omega \subset \mathbb{R}^{n}$, it is necessary to contemplate the "boundary condition" not only on $\partial \Omega$ but in the whole $\mathbb{R}^{n} \backslash \Omega$. The natural space to work with is denoted as $H_{0}^{s}(\Omega)$ and it is defined by the closure of $C_{c}^{\infty}(\Omega)$ in the norm $\|\cdot\|_{s}$. When $\Omega$ is a Lipschitz domain, $H_{0}^{s}(\Omega)$ coincides with the space of functions vanishing outside $\Omega$, i.e.,

$$
H_{0}^{s}(\Omega)=\left\{u \in H^{s}\left(\mathbb{R}^{n}\right): u=0 \text { in } \mathbb{R}^{n} \backslash \Omega\right\} .
$$

Aimed at our purposes in this paper, it is suitable to analyze the behavior of the normalization constant $c(n, s)$ as $s \uparrow 1$. In [35], E. Stein studied the relation between negative powers of the Laplace operator and Riesz potentials. In this context it is proved that

$$
\lim _{s \uparrow 1} \frac{c(n, s)}{1-s}=\frac{4 n}{\omega_{n-1}},
$$

where $\omega_{n-1}$ denotes the $n-1$-dimensional measure of the unit sphere $S^{n-1}$. That choise of the constant is consistent in order to recover the usual laplacian in the sense that

$$
\lim _{s \uparrow 1}(-\Delta)^{s} u=-\Delta u \quad \forall u \in C_{c}^{\infty}\left(\mathbb{R}^{n}\right) .
$$

For a direct proof of these facts, we refer to the article [13].

Moreover, in [13, Remark 4.3] is shown that

$$
\lim _{s \uparrow 1} \frac{c(n, s)}{2}[u]_{s}^{2}=\|\nabla u\|_{2}^{2} .
$$

2.2. Statements of the main results. We begin with some definitions.

Definition 2.1. Let $\Omega \subset \mathbb{R}^{n}$ be an open set. Given $A \subset \Omega$, for any $0<s<1$, we define the Gagliardo $s$-capacity of $A$ relative to $\Omega$ as

$$
\operatorname{cap}_{s}(A, \Omega)=\inf \left\{[u]_{s}^{2}: u \in C_{c}^{\infty}(\Omega), u \geq 0, A \subset\{u \geq 1\}^{\circ}\right\} .
$$

In this context, we say that a subset $A$ of $\Omega$ is a $s$-quasi open set if there exists a decreasing sequence $\left\{\omega_{k}\right\}_{k \in \mathbb{N}}$ of open subsets of $\Omega$ such that $\operatorname{cap}_{s}\left(\omega_{k}, \Omega\right) \rightarrow 0$, as $k \rightarrow \infty$, and $A \cup \omega_{k}$ is an open set for all $k \in \mathbb{N}$. 
We denote by $\mathcal{A}_{s}(\Omega)$ the class of all $s$-quasi open subsets of $\Omega$.

In the case $s=1$ the definitions are completely analogous with $\|\nabla u\|_{2}$ instead of $[u]_{s}^{2}$.

Remark 2.2. From Hölder's inequality is easy to see that $\mathcal{A}_{s}(\Omega) \subset \mathcal{A}_{t}(\Omega)$ when $0<t<s \leq 1$.

For further properties of the $s$-capacity we refer the reader, for instance, to [32].

Given $A \in \mathcal{A}_{s}(\Omega)$, we denote by $u_{A}^{s} \in H_{0}^{s}(A)$ the unique (weak) solution to

$$
(-\Delta)^{s} u_{A}^{s}=1 \quad \text { in } A, \quad u_{A}^{s}=0 \quad \text { in } \mathbb{R}^{n} \backslash A .
$$

Remark 2.3. Observe also that $u_{A}^{s}$ is the unique minimizer of

$$
I_{s}(u):=\frac{c(n, s)}{2}[u]_{s}^{2}-\int_{A} u d x
$$

in $H_{0}^{s}(A)$.

With this notation, we define the following notion of set convergence.

Definition 2.4. Let $\left\{A_{k}\right\}_{k \in \mathbb{N}} \subset \mathcal{A}_{s}(\Omega)$ and $A \in \mathcal{A}_{s}(\Omega)$. We say that $A_{k} \stackrel{\gamma_{s}}{\rightarrow} A$ if $u_{A_{k}}^{s} \rightarrow u_{A}^{s}$ strongly in $L^{2}(\Omega)$.

Remark 2.5. This is the fractional version of the $\gamma$-convergence of sets defined in [9].

Now, take $0<s<1$ be fixed and let $F_{s}: \mathcal{A}_{s}(\Omega) \rightarrow \mathbb{R}$ be such that

$\left(H_{1}^{s}\right) F_{s}$ is lower semicontinuous with respect to the $\gamma_{s}$-convergence; that is,

$$
A_{k} \stackrel{\gamma_{s}}{\rightarrow} A \text { implies } \quad F_{s}(A) \leq \liminf _{k \rightarrow \infty} F_{s}\left(A_{k}\right) .
$$

$\left(H_{2}^{s}\right) F_{s}$ is decreasing with respect to set inclusion; that is $F_{s}(A) \geq F_{s}(B)$ whenever $A \subset B$.

So, the problem that we address in this paper is the following:

$$
\min \left\{F_{s}(A): A \in \mathcal{A}_{s}(\Omega),|A| \leq c\right\},
$$

where $F_{s}$ satisfies $\left(H_{1}^{s}\right)-\left(H_{2}^{s}\right)$.

Remark 2.6. Observe that from the monotonicity assumption $\left(H_{2}^{s}\right)$ on $F_{s}$, this problem is equivalent to minimize in the class of $s$-quasi open sets $A$ of measure $|A|=c$. In fact, assume that a minimizer $A_{0} \in \mathcal{A}_{s}$ for (2.6) verifies that $\left|A_{0}\right|<c$. Then, for any $\tilde{A}_{0} \supset A_{0}$ such that $\left|\tilde{A}_{0}\right|=c$ we have

$$
F_{s}\left(\tilde{A}_{0}\right) \leq F_{s}\left(A_{0}\right)=\inf _{A \in \mathcal{A}_{s}} F_{s}(A)
$$

and so $\tilde{A}_{0}$ is a minimizer of $F_{s}$.

Following the same approach and ideas of [9], problem (2.6) can be analyzed and that is the content of our first result. 
Theorem 2.7. Let $0<s<1$ be fixed and $\Omega \subset \mathbb{R}^{n}$ be open and bounded. Let $F_{s}: \mathcal{A}_{s}(\Omega) \rightarrow \mathbb{R}$ be such that $\left(H_{1}^{s}\right)$ and $\left(H_{2}^{s}\right)$ are satisfied.

Then, for every $0<c<|\Omega|$, problem (2.6) has a solution.

As we mentioned, the proof of Theorem 2.7 follows the ideas developed in [9] and that is carried out in Section 3 .

Next, we want to analyze the behavior of these minimum problems and its minimizers when $s \uparrow 1$.

In order to perform such analysis we need to assume some asymptotic behavior on the cost functionals $F_{s}$. In order to do this, we need to define a notion of convergence for sets when $s$ varies.

Definition 2.8. Let $0<s_{k} \uparrow 1$ and let $A_{k} \in \mathcal{A}_{s_{k}}(\Omega)$ and $A \in \mathcal{A}_{1}(\Omega)$. We say that $A_{k} \stackrel{\gamma}{\rightarrow} A$ if $u_{A_{k}}^{s_{k}} \rightarrow u_{A}^{1}$ strongly in $L^{2}(\Omega)$.

Remark 2.9. Observe that the notion of $\gamma$-convergence of sets given in [9] is denoted in this paper by $\gamma_{1}$-convergence. This should not cause any confusion.

Now we can give the assumptions of the functionals $F_{s}$ :

$\left(H_{1}\right)$ Continuity with respect to $A$; that is, if $A \in \mathcal{A}_{1}(\Omega)$, then

$$
F_{1}(A)=\lim _{s \uparrow 1} F_{s}(A) .
$$

$\left(H_{2}\right)$ Liminf inequality; that is, for every $0<s_{k} \uparrow 1$ and $A_{k} \stackrel{\gamma}{\rightarrow} A$, then

$$
F_{1}(A) \leq \liminf _{k \rightarrow \infty} F_{s_{k}}\left(A_{k}\right)
$$

Under these assumptions, we obtain the following result.

Theorem 2.10. For any $0<s \leq 1$, let $F_{s}: \mathcal{A}_{s}(\Omega) \rightarrow \mathbb{R}$ be such that $\left(H_{1}^{s}\right)$ and $\left(H_{2}^{s}\right)$ are satisfied. Assume moreover that $\left(H_{1}\right)$ and $\left(H_{2}\right)$ are satisfied.

Then

$$
\min \left\{F_{1}(A): A \in \mathcal{A}_{1}(\Omega),|A| \leq c\right\}=\lim _{s \uparrow 1} \min \left\{F_{s}(A): A \in \mathcal{A}_{s}(\Omega),|A| \leq c\right\}
$$

and, moreover, if $A_{s} \in \mathcal{A}_{s}(\Omega)$ is a minimizer for (2.6), then there exists a sequence $0<s_{k} \uparrow 1$, sets $\tilde{A}_{s_{k}} \supset A_{s_{k}}$ and a set $A_{1} \in \mathcal{A}_{1}(\Omega)$ such that $\tilde{A}_{s_{k}} \stackrel{\gamma}{\rightarrow} A_{1}$ and $A_{1}$ is a minimizer for (2.6) with $s=1$.

The proof of Theorem 2.10 is carried out in Section 4 and also uses ideas developed in [9]. However, in this case nontrivial modifications need to be made in order to consider the varying spaces where one is working.

2.3. Examples. Let first establish some notations. Given a bounded domain $A \in$ $\mathcal{A}_{s}(\Omega)$, consider the problem

$$
(-\Delta)^{s} u=\lambda^{s} u \quad \text { in } A, \quad u \in H_{0}^{s}(A)
$$

where $\lambda^{s} \in \mathbb{R}$ is the eigenvalue parameter. It is well-known that there exists a discrete sequence $\left\{\lambda_{k}^{s}(A)\right\}_{k \in \mathbb{N}}$ of positive eigenvalues of (2.7) approaching $+\infty$ whose corresponding eigenfunctions $\left\{u_{k}^{s}\right\}_{k \in \mathbb{N}}$ form an orthogonal basis in $L^{2}(A)$. These facts follows directly from the spectral theorem for compact and self adjoints 
operators, see [6]. Moreover, the following variational characterization holds for the eigenvalues

$$
\lambda_{k}^{s}(A)=\min _{u \perp W_{k-1}} \frac{c(n, s)}{2} \frac{[u]_{s}^{2}}{\|u\|_{2}^{2}},
$$

where $W_{k}$ is the space spanned by the first $k$ eigenfunctions $u_{1}^{s}, \ldots, u_{k}^{s}$.

Functions $F_{s}$ satisfying hypothesis $\left(H_{1}^{s}\right)$ and $\left(H_{2}^{s}\right)$ include a large family of examples. For instance, if we consider the application $A \mapsto \lambda_{k}^{s}(A)$, Theorem 2.7 claims that for every $k \in \mathbb{N}$ and $0<c<|\Omega|$, the minimum

$$
\min \left\{\lambda_{k}^{s}(A): A \in \mathcal{A}_{s}(\Omega),|A| \leq c\right\}
$$

is achieved. More generally, the minimum

$$
\min \left\{\Phi_{s}\left(\lambda_{k_{1}}^{s}(A), \ldots, \lambda_{k_{N}}^{s}(A)\right): A \in \mathcal{A}_{s}(\Omega),|A| \leq c\right\}
$$

is achieved, where $\Phi_{s}: \mathbb{R}^{N} \rightarrow \overline{\mathbb{R}}$ is lower semicontinuous and increasing in each coordinate.

Moreover, if $\Phi_{s}\left(t_{1}, \ldots, t_{N}\right) \rightarrow \Phi_{1}\left(t_{1}, \ldots, t_{N}\right)$ for every $\left(t_{1}, \ldots, t_{N}\right) \in \mathbb{R}^{N}$ and

$$
\Phi_{1}\left(t_{1}, \ldots, t_{N}\right) \leq \liminf _{k \rightarrow \infty} \Phi_{s_{k}}\left(t_{1}^{k}, \ldots, t_{N}^{k}\right),
$$

for every $\left(t_{1}^{k}, \ldots, t_{N}^{k}\right) \rightarrow\left(t_{1}, \ldots, t_{N}\right)$, then Theorem 2.10 together with the result of [5] imply that

$$
\begin{aligned}
\min \left\{\Phi_{1}\right. & \left.\left(\lambda_{k_{1}}(A), \ldots, \lambda_{k_{N}}(A)\right): A \in \mathcal{A}_{1}(\Omega),|A| \leq c\right\} \\
& =\lim _{s \uparrow 1} \min \left\{\Phi_{s}\left(\lambda_{k_{1}}^{s}(A), \ldots, \lambda_{k_{N}}^{s}(A)\right): A \in \mathcal{A}_{s}(\Omega),|A| \leq c\right\} .
\end{aligned}
$$

\section{Proof of Theorem 2.7}

This section is devoted to prove Theorem 2.7. The arguments follow essentially the lines of [9] with some modifications for the nonlocal setting.

The sketch of the argument is as follows: Given $A \in \mathcal{A}_{s}(\Omega)$, we first prove that $u_{A}^{s}$ is the solution to

$$
\max \left\{w \in H_{0}^{s}(\Omega): w \leq 0 \text { in } \mathbb{R}^{n} \backslash A,(-\Delta)^{s} w \leq 1 \text { in } \Omega\right\} .
$$

Moreover, as a consequence of Lemma 3.2 below, $u_{A}^{s}$ belongs to the convex closed set $\mathcal{K}_{s}$ defined as

$$
\mathcal{K}_{s}=\left\{w \in H_{0}^{s}(\Omega): w \geq 0,(-\Delta)^{s} w \leq 1 \text { in } \Omega\right\} .
$$

It will be convenient to also consider $\mathcal{K}_{1}$ defined as in (3.2) with $s=1$ where $(-\Delta)^{1}=-\Delta$.

Finally, one defines a functional $G_{s}$ on $\mathcal{K}_{s}$ satisfying that

$\left(G_{1}\right) G_{s}$ is decreasing on $\mathcal{K}_{s}$,

$\left(G_{2}\right) G_{s}$ is lower semicontinuous on $\mathcal{K}_{s}$ with respect to the strong topology on $L^{2}(\Omega)$

$\left(G_{3}\right) G_{s}\left(u_{A}^{s}\right)=F_{s}(A)$ for every $A \in \mathcal{A}_{s}(\Omega)$,

to conclude that the problem

$$
\min \left\{G_{s}(w): w \in \mathcal{K}_{s},|\{w>0\}| \leq c\right\}
$$


has a solution $w_{0}$. If we denote by $A_{0}=\left\{w_{0}>0\right\}$, then $u_{A_{0}}^{s}$ is also a minimum point of $G_{s}$ over the whole $\mathcal{K}_{s}$ subject to the condition $|\{w>0\}| \leq c$ and hence, $A_{0}$ is a minimizer for $F_{s}$ in $\mathcal{A}_{s}(\Omega)$ subject to the condition $|A| \leq c$.

We start by proving (3.1). Let us define

$$
K_{A}=\left\{w \in H_{0}^{s}(\Omega): w \leq 0 \text { in } \mathbb{R}^{n} \backslash A\right\},
$$

and $w_{A} \in K_{A}$ the (unique) minimizer of

$$
I_{s}: K_{A} \rightarrow \mathbb{R}, \quad I_{s}(w)=\frac{c(n, s)}{2}[w]_{s}^{2}-\int_{\Omega} w d x .
$$

Observe that, by Stampacchia's Theorem, $w_{A}$ is characterized by the variational inequality

$$
\mathcal{E}\left(w_{A}, v-w_{A}\right) \geq \int_{\Omega}\left(v-w_{A}\right) d x \quad \forall v \in K_{A},
$$

where we denote

$$
\mathcal{E}(u, v):=c(n, s) \iint_{\mathbb{R}^{n} \times \mathbb{R}^{n}} \frac{(u(x)-u(y))(v(x)-v(y))}{|x-y|^{n+2 s}} d x d y .
$$

Next, we prove that both functions $u_{A}^{s}$ and $w_{A}$ agree.

Lemma 3.1. With the previous notation we have that $w_{A}=u_{A}^{s}$.

Proof. The proof is standard. We will use the standard notations of $w^{+}=\max \{w, 0\}$ and $w^{-}=\max \{-w, 0\}$.

Take $w_{A}^{+}$as test function in the variational inequality (3.4) and obtain

$$
\begin{aligned}
0 \leq \int_{\Omega} w_{A}^{-} d x & \leq \mathcal{E}\left(w_{A}, w_{A}^{-}\right) \\
& \leq-c(n, s) \iint_{\left\{w_{A} \leq 0\right\} \times\left\{w_{A} \leq 0\right\}} \frac{\left(w_{A}^{-}(x)-w_{A}^{-}(y)\right)^{2}}{|x-y|^{n+2 s}} d x d y .
\end{aligned}
$$

From this inequality one easily conclude that $w_{A}^{-}=0$ and so, since $w_{A} \in K_{A}$, $w_{A} \in H_{0}^{s}(A)$.

Therefore, since, by Remark 2.3, $u_{A}^{s}$ is the unique minimum of $I_{s}$ over $H_{0}^{s}(A)$ and, since also $u_{A}^{s} \in K_{A}, I_{s}\left(w_{A}\right) \leq I_{s}\left(u_{A}^{s}\right)$ the lemma follows.

Using the previous lemma we prove the following properties on $u_{A}^{s}$.

Lemma 3.2. With the above notations, $u_{A}^{s} \geq 0$ on $\Omega$. Moreover, $u_{A}^{s}$ is the solution to $(3.1)$.

Proof. First observe that from the maximum principle it follows that $u_{A}^{s} \geq 0$ in $\Omega$.

Given $v \in H_{0}^{s}(\Omega)$ such that $v \geq 0$, we have that $-v \in K_{A}$. Using it as a test function in (3.4) we obtain that

$$
\mathcal{E}\left(u_{A}^{s},-v-u_{A}^{s}\right)=-c(n, s)\left[u_{A}^{s}\right]_{s}^{2}-\mathcal{E}\left(u_{A}^{s}, v\right) \geq-\int_{\Omega} v d x-\int_{\Omega} u_{A}^{s} d x .
$$

Using that $(-\Delta)^{s} u_{A}^{s}=1$ in $A$, the last inequality reads as

$$
\mathcal{E}\left(u_{A}^{s}, v\right) \leq \int_{\Omega} v d x
$$


Since $v \in H_{0}^{s}(\Omega)$ is nonnegative but otherwise arbitrary, we get that $(-\Delta)^{s} u_{A}^{s} \leq 1$ in $\Omega$.

Finally, if $w \leq 0$ in $\mathbb{R}^{n} \backslash A$ and $(-\Delta)^{s} w \leq 1$ in $\Omega$, then

$$
(-\Delta)^{s} w \leq(-\Delta)^{s} u_{A}^{s} \text { in } A \text { and } w \leq u_{A}^{s} \text { in } \mathbb{R}^{n} \backslash A .
$$

Hence, by comparison, $w \leq u_{A}^{s}$ in $\mathbb{R}^{n}$.

The set $\mathcal{K}_{s}$ satisfies the following properties:

Proposition 3.3. $\mathcal{K}_{s}$ is a convex, closed and bounded subset of $H_{0}^{s}(\Omega)$.

Proof. Clearly, $\mathcal{K}_{s}$ is a convex set. $\mathcal{K}_{s}$ is also bounded. Indeed, given $u \in \mathcal{K}_{s}$, by Hölder and Poincaré's inequalities we get

$$
c(n, s)[u]_{s}^{2} \leq \int_{\Omega} u d x \leq|\Omega|^{\frac{1}{2}}\|u\|_{L^{2}(\Omega)} \leq C|\Omega|^{\frac{1}{2}}[u]_{s} .
$$

In order to see that $\mathcal{K}_{s}$ is closed, let $\left\{u_{k}\right\}_{k \in \mathbb{N}}$ be a sequence in $\mathcal{K}_{s}$ such that $u_{k} \rightarrow u$ in $H_{0}^{s}(\Omega)$. For any $k \in \mathbb{N}$ and any $v \in H_{0}^{s}(\Omega), v \geq 0$, it holds that

$$
\mathcal{E}\left(u_{k}, v\right) \leq \int_{\Omega} v d x
$$

Since $\mathcal{E}(\cdot, v)$ is continuous in $H_{0}^{s}(\Omega)$ (in fact is weakly continuous), taking the limit as $k \rightarrow \infty$ we obtain that $\mathcal{E}(u, v) \leq \int_{\Omega} v d x$, but, since $v \in H_{0}^{s}(\Omega)$ is nonnegative but otherwise arbitrary we obtain that $(-\Delta)^{s} u \leq 1$ in $\Omega$ and then $u \in \mathcal{K}_{s}$.

Remark 3.4. Observe that optimal constant in Poincaré's inequality

$$
\|u\|_{L^{2}(\Omega)}^{2} \leq C(\Omega, s)[u]_{s}^{2},
$$

has a dependence on $s$ of the form

$$
C(\Omega, s) \leq(1-s) C(\Omega) .
$$

See [5].

Therefore, the proof of Proposition 3.3 gives that if $u \in \mathcal{K}_{s}$, then

$$
(1-s)[u]_{s}^{2} \leq C
$$

where $C$ depends on $\Omega$ but is independent on $0<s<1$.

Now, in order to prove the existence of solution to (2.6) we define a functional $G_{s}$ on $\mathcal{K}_{s}$ satisfying the conditions $\left(G_{1}\right)-\left(G_{3}\right)$.

We will use the notation, for $0<s \leq 1$,

$$
\mathcal{A}_{s}^{c}(\Omega):=\left\{A \in \mathcal{A}_{s}(\Omega):|A| \leq c\right\} .
$$

For any $0<s \leq 1$, given $w \in \mathcal{K}_{s}$ we define

$$
J_{s}(w)=\inf \left\{F_{s}(A): A \in \mathcal{A}_{s}^{c}(\Omega), u_{A}^{s} \leq w\right\} .
$$

This functional $J_{s}$ is not lower semicontinuous in general. So we define $G_{s}$ to be the lower semicontinuos envelope of $J_{s}$ in $\mathcal{K}_{s}$ with respect to the strong topology in $L^{2}(\Omega)$, i.e.,

$$
G_{s}(w)=\inf \left\{\liminf _{k \rightarrow \infty} J_{s}\left(w_{k}\right)\right\},
$$

where the infimum is taken over all sequences $\left\{w_{k}\right\}_{k \in \mathbb{N}}$ in $\mathcal{K}_{s}$ such that $w_{k} \rightarrow w$ in $L^{2}(\Omega)$. 
Observe that $G_{s}$ automatically verifies $\left(G_{2}\right)$.

Proposition 3.5. Let $0<s \leq 1$. The functional $G_{s}$ satisfies conditions $\left(G_{1}\right)$.

Proof. The case $s=1$ is considered in [9], so we take $0<s<1$.

Let us begin by noticing that if $u, v \in \mathcal{K}_{s}$, then $\max \{u, v\} \in \mathcal{K}_{s}$. In fact, let us denote $w=\max \{u, v\}$ and consider the convex set

$$
E=\left\{z \in H_{0}^{s}(\Omega): z \leq w \text { in } \Omega\right\} .
$$

By Stampacchia's Theorem there exists a unique function $z_{0} \in E$ such that

$$
I_{s}\left(z_{0}\right)=\min _{E} I_{s}
$$

where $I_{s}$ is defined in (2.5).

In addition, $z_{0}$ satisfies that

$$
\mathcal{E}\left(z_{0}, z-z_{0}\right) \geq \int_{\Omega}\left(z-z_{0}\right) d x \quad \text { for all } z \in E,
$$

where $\mathcal{E}$ is defined in (3.5).

Let us see that $(-\Delta)^{s} z_{0} \leq 1$. Given $\varphi \in H_{0}^{s}(\Omega)$ such that $\varphi \leq 0$, we define the functional

$$
i(t)=I_{s}\left(z_{0}+t \varphi\right), \text { for all } t \geq 0 .
$$

Observe that $i^{\prime}(0) \geq 0$. In consequence, for any non-positive $\varphi \in H_{0}^{s}(\Omega)$ it holds that $\mathcal{E}\left(z_{0}, \varphi\right) \geq \int_{\Omega} \varphi d x$, and then $\mathcal{E}(z, \varphi) \leq \int_{\Omega} \varphi d x$ for any $\varphi \in H_{0}^{s}(\Omega), \varphi \geq 0$ and the claim follows.

Now, we will prove that $z_{0} \geq u$ (and for symmetry reasons that $z_{0} \geq v$ ), from where it will follow that $z_{0} \geq w$. Since $z_{0} \in E$, the reverse inequality holds and we can conclude that $z_{0}=w \in \mathcal{K}_{s}$.

Let $\eta=\max \left\{z_{0}, u\right\}$ and let us see that $z_{0}=\eta$. Observe that $\eta \in E$ and thus it can be consider as a test function in (3.10). Thus,

$$
\mathcal{E}\left(z_{0}, \eta-z_{0}\right) \geq \int_{\Omega}\left(\eta-z_{0}\right) d x .
$$

On the other hand, since $\eta-z_{0} \geq 0$ and $(-\Delta)^{s} u \leq 1$ in $\Omega$, it follows that

$$
\mathcal{E}\left(u, \eta-z_{0}\right) \leq \int_{\Omega}\left(\eta-z_{0}\right) d x .
$$

From both inequalities it is straightforward to see that

$$
0 \leq \mathcal{E}\left(z_{0}-u, \eta-z_{0}\right) \leq-c(n, s)\left[\left(u-z_{0}\right)^{+}\right]_{s}^{2}
$$

and then $\left(u-z_{0}\right)^{+}=0$ in $\mathbb{R}^{n}$, which implies that $z_{0} \geq u$ in $\mathbb{R}^{n}$, as we required.

Now we proceed with the proof.

Let $u, v \in \mathcal{K}_{s}$ be such that $u \leq v$ and let $\left\{u_{k}\right\}_{k \in \mathbb{N}} \subset \mathcal{K}_{s}$ be such that $u_{k} \rightarrow u$ in $L^{2}(\Omega)$ and $J_{s}\left(u_{k}\right) \rightarrow G_{s}(u)$.

By our previous claim, $v_{k}=\max \left\{v, u_{k}\right\} \in \mathcal{K}_{s}$ for each $k \in \mathbb{N}$ and $v_{k} \rightarrow v=$ $\max \{v, u\}$ in $L^{2}(\Omega)$. Consequently, since $J_{s}$ is non-increasing and $v_{k} \geq u_{k}$ for any $k \in \mathbb{N}$ we get

as we wanted to show.

$$
G_{s}(v) \leq \liminf _{k \rightarrow \infty} J_{s}\left(v_{k}\right) \leq \lim _{k \rightarrow \infty} J_{s}\left(u_{k}\right)=G_{s}(u),
$$


We will need the following lemma in order to prove condition $\left(G_{3}\right)$. We omit the proof since it is completely analogous to that of Lemmas 3.2 and 3.3 in [9] where the case $s=1$ was considered.

Lemma 3.6. Let $0<s<1$. Let $\left\{A_{k}\right\}_{k \in \mathbb{N}} \subset \mathcal{A}_{s}(\Omega)$ be such that $u_{A_{k}}^{s} \rightarrow u$ in $L^{2}(\Omega)$, with $u \leq u_{A}^{s}$. We define $A^{\varepsilon}=\left\{u_{A}^{s}>\varepsilon\right\}$. Then, if $u_{A_{k} \cup A^{\varepsilon}}^{s} \rightarrow u^{\varepsilon}$ in $L^{2}(\Omega)$ it holds that $u^{\varepsilon} \leq u_{A}^{s}$.

With the help of Lemma 3.6 we are able to show that $G_{s}$ satisfies condition $\left(G_{3}\right)$.

Proposition 3.7. Let $0<s \leq 1$. Then the functional $G_{s}$ satisfies $\left(G_{3}\right)$.

Proof. We only need to consider $0<s<1$. Let us fix $A \in \mathcal{A}_{s}^{c}(\Omega)$. From (3.9) it follows that $G_{s}\left(u_{A}^{s}\right) \leq F_{s}(A)$. To prove the reverse inequality, it suffices to see that

$$
F_{s}(A) \leq \liminf _{k \rightarrow \infty} J_{s}\left(w_{k}\right)
$$

for any sequence $\left\{w_{k}\right\}_{k \in \mathbb{N}} \subset \mathcal{K}_{s}$ such that $w_{k} \rightarrow u_{A}^{s}$ in $L^{2}(\Omega)$.

By definition of $J_{s}$, there exists $A_{k} \in \mathcal{A}_{s}^{c}(\Omega)$ such that

$$
F_{s}\left(A_{k}\right) \leq J_{s}\left(w_{k}\right)+\frac{1}{k} \quad \text { and } \quad u_{A_{k}}^{s} \leq w_{k} .
$$

Observe that $u_{A_{k}}^{s} \in \mathcal{K}_{s}$ for each $k \in \mathbb{N}$ and by Proposition 3.3, $\left\{u_{A_{k}}^{s}\right\}_{k \in \mathbb{N}}$ is bounded in $H_{0}^{s}(\Omega)$. Then, up to a subsequence, there exists $u \in \mathcal{K}_{s}$ such that $u_{A_{k}}^{s} \rightarrow u$ in $L^{2}(\Omega)$. Since $w_{k} \rightarrow u_{A}^{s}$ in $L^{2}(\Omega)$, from $u_{A_{k}}^{s} \leq w_{k}$ we get $u \leq u_{A}^{s}$.

Let us consider the set $A^{\varepsilon}=\left\{u_{A}^{s}>\varepsilon\right\}$ and observe that $u_{A_{k} \cup A^{\varepsilon}}^{s} \in \mathcal{K}_{s}$. Again by Proposition 3.3, it follows that and $u_{A_{k} \cup A^{\varepsilon}}^{s} \rightarrow u^{\varepsilon}$ in $L^{2}(\Omega)$ for some $u^{\varepsilon} \in \mathcal{K}_{s}$. By Lemma 3.6, inequality $u^{\varepsilon} \leq u_{A}^{s}$ follows.

We claim that $\left(u_{A}^{s}-\varepsilon\right)^{+} \leq u_{A^{\varepsilon}}^{s}$. Indeed,

$$
\left(u_{A}^{s}-\varepsilon\right)^{+}(x)-\left(u_{A}^{s}-\varepsilon\right)^{+}(y)= \begin{cases}u_{A}^{s}(x)-u_{A}^{s}(y) & \text { if } x, y \in A^{\varepsilon} \\ u_{A}^{s}(x)-\varepsilon & \text { if } x \in A^{\varepsilon} \text { and } y \notin A^{\varepsilon} \\ -u_{A}^{s}(y)+\varepsilon & \text { if } x \notin A^{\varepsilon} \text { and } y \in A^{\varepsilon} \\ 0 & \text { otherwise. }\end{cases}
$$

Then, for any $v \in H_{0}^{s}\left(A^{\varepsilon}\right)$ such that $v \geq 0$, we get

$$
\begin{aligned}
& \iint_{\mathbb{R}^{n} \times \mathbb{R}^{n}} \frac{\left(\left(u_{A}^{s}(x)-\varepsilon\right)^{+}-\left(\left(u_{A}^{s}(y)-\varepsilon\right)^{+}\right)(v(x)-v(y))\right.}{|x-y|^{n+2 s}} d x d y= \\
& \iint_{A^{\varepsilon} \times A^{\varepsilon}} \frac{\left(u_{A}^{s}(x)-u_{A}^{s}(y)\right)(v(x)-v(y))}{|x-y|^{n+2 s}} d x d y+2 \iint_{A^{\varepsilon} \times\left(A^{\varepsilon}\right)^{c}} \frac{\left(u_{A}^{s}(x)-\varepsilon\right) v(x)}{|x-y|^{n+2 s}} d y d x= \\
& \iint_{\mathbb{R}^{n} \times \mathbb{R}^{n}} \frac{\left(u_{A}^{s}(x)-u_{A}^{s}(y)\right)(v(x)-v(y))}{|x-y|^{n+2 s}} d x d y+2 \iint_{A^{\varepsilon} \times\left(A^{\varepsilon}\right)^{c}} \frac{\left(u_{A}^{s}(y)-\varepsilon\right) v(x)}{|x-y|^{n+2 s}} d y d x \leq \\
& \iint_{\mathbb{R}^{n} \times \mathbb{R}^{n}} \frac{\left(u_{A}^{s}(x)-u_{A}^{s}(y)\right)(v(x)-v(y))}{|x-y|^{n+2 s}} d x d y
\end{aligned}
$$

That is, $(-\Delta)^{s}\left(u_{A}^{s}-\varepsilon\right)^{+} \leq(-\Delta)^{s} u_{A}^{s}=1=\left(-\Delta^{s}\right) u_{A^{\varepsilon}}^{s}$ in $A^{\varepsilon}$. Moreover, since $0=\left(u_{A}^{s}-\varepsilon\right)^{+}=u_{A^{\varepsilon}}^{s}$ in $\mathbb{R}^{n} \backslash A^{\varepsilon}$, from the comparison principle it follows that $\left(u_{A}^{s}-\varepsilon\right)^{+} \leq u_{A^{\varepsilon}}^{s}$ in $\mathbb{R}^{n}$. 
We have obtained the following chain of inequalities

$$
\left(u_{A}^{s}-\varepsilon\right)^{+} \leq u_{A^{\varepsilon}}^{s} \leq u_{A_{k} \cup A^{\varepsilon}}^{s} .
$$

Taking limit as $k \rightarrow \infty$ we conclude that

$$
\left(u_{A}^{s}-\varepsilon\right)^{+} \leq u^{\varepsilon} \leq u_{A}^{s} .
$$

since $u^{\varepsilon} \leq u_{A}^{s}$ and $u_{A_{k} \cup A^{\varepsilon}}^{s} \rightarrow u^{\varepsilon}$.

Since $u^{\varepsilon} \in \mathcal{K}_{s}$, by (3.6), $\left\{u^{\varepsilon}\right\}_{\varepsilon>0}$ is uniformly bounded in $H_{0}^{s}(\Omega)$. Consequently, up to a subsequence, $u^{\varepsilon} \rightarrow u_{A}^{s} \in L^{2}(\Omega)$.

By a standard diagonal argument, there exists a sequence $\left\{\varepsilon_{k}\right\}_{k \in \mathbb{N}}$ such that $u_{A_{k} \cup A^{\varepsilon} k}^{s} \rightarrow u_{A}^{s}$ in $L^{2}(\Omega)$.

In conclusion, we have proved that $\left(A_{k} \cup A^{\varepsilon_{k}}\right) \gamma_{s}$-converges to $A$. Therefore

$$
F_{s}(A) \leq \liminf _{k \rightarrow \infty} F_{s}\left(A_{k} \cup A^{\varepsilon_{k}}\right) \leq \liminf _{k \rightarrow \infty} F_{s}\left(A_{k}\right) \leq \liminf _{k \rightarrow \infty} J_{s}\left(w_{k}\right) .
$$

This fact concludes the proof of the proposition.

Having proved these preliminary results, the proof of Theorem 2.7 follows in the same way of that of [9, Theorem 2.5]. We include the details for the reader convenience.

Proof of Theorem 2.7. First, we solve (3.3). Take $\left\{w_{k}\right\}_{k \in \mathbb{N}} \subset \mathcal{K}_{s}$ be such that $\left|\left\{w_{k}>0\right\}\right| \leq c$ and

$$
\lim _{k \rightarrow \infty} G_{s}\left(w_{k}\right)=\inf \left\{G_{s}(w): w \in \mathcal{K}_{s},|\{w>0\}| \leq c\right\}=: m_{G_{s}} .
$$

By Proposition 3.3, there exists $w_{0} \in \mathcal{K}_{s}$ such that $w_{k} \rightarrow w_{0}$ strongly in $L^{2}(\Omega)$, up to a subsequence. Thus, $\left|\left\{w_{0}>0\right\}\right| \leq c$. Then, by $\left(G_{2}\right)$,

$$
m_{G_{s}} \leq G_{s}\left(w_{0}\right) \leq \liminf _{k \rightarrow \infty} G_{s}\left(w_{k}\right)=m_{G_{s}} .
$$

So, $w_{0}$ is a solution to (3.3).

Now, consider $A_{0}:=\left\{w_{0}>0\right\}$. Then, $A_{0} \in \mathcal{A}_{s}^{c}(\Omega)$. By Lemma 3.2, $w_{0} \leq u_{A_{0}}^{s}$.

For every $A \in \mathcal{A}_{s}^{c}(\Omega)$, we konw that $u_{A}^{s} \in \mathcal{K}_{s},\left|\left\{u_{A}^{s}>0\right\}\right| \leq c$. Then, by $\left(G_{3}\right),\left(G_{1}\right)$ and the fact that $w_{0}$ is the solution to (3.3), we have

$$
F_{s}\left(A_{0}\right)=G_{s}\left(u_{A_{0}}^{s}\right) \leq G_{s}\left(w_{0}\right) \leq G_{s}\left(u_{A}^{s}\right)=F_{s}(A) .
$$

Therefore, $A_{0}$ is a solution to (2.6).

\section{Proof of Theorem 2.10}

In this section we prove Theorem 2.10 following the same spirit of [9], however, nontrivial changes must be performed due to the nonlocal settings.

Our first goal is to show that a sequence $\left\{u_{k}\right\}_{k \in \mathbb{N}} \subset L^{2}(\Omega)$ such that $u_{k} \in \mathcal{K}_{s_{k}}$ is precompact and that every accumulation point belongs to $\mathcal{K}_{1}$.

This is the content of the next lemma.

Lemma 4.1. Let $0<s_{k} \uparrow 1$ and let $u_{k} \in \mathcal{K}_{s_{k}}$. Then, there exists $u \in H_{0}^{1}(\Omega)$ and a subsequence $\left\{u_{k_{j}}\right\}_{j \in \mathbb{N}} \subset\left\{u_{k}\right\}_{k \in \mathbb{N}}$ such that $u_{k_{j}} \rightarrow u$ strongly in $L^{2}(\Omega)$.

Moreover, if $u_{k} \in \mathcal{K}_{s_{k}}$ is such that $u_{k} \rightarrow u$ strongly in $L^{2}(\Omega)$, then $u \in \mathcal{K}_{1}$. 
Proof. From Remark 3.4, there exists a constant $C>0$ such that

$$
\sup _{k \in \mathbb{N}}\left(1-s_{k}\right)\left[u_{k}\right]_{s_{k}}^{2} \leq C .
$$

Now the first claim follows from [3, Theorem 4].

Now, assume that $u_{k} \rightarrow u$ in $L^{2}(\Omega)$. It is clear that $u \geq 0$. Since $(-\Delta)^{s_{k}} u_{k} \leq 1$ in $\Omega$, for every non-negative $\varphi \in C_{c}^{\infty}(\Omega)$ we have that

$$
\int_{\Omega}(-\Delta)^{s_{k}} \varphi u_{k} d x=\left\langle(-\Delta)^{s_{k}} u_{k}, \varphi\right\rangle \leq \int_{\Omega} \varphi d x .
$$

By the convergence assumption on $u_{k}$ and the fact that the convergence (2.2) is also strong in $L^{2}(\Omega)$, we can take limit as $k \rightarrow \infty$ in the previous inequality to obtain that

$$
\int_{\Omega}-\Delta \varphi u d x=\langle-\Delta u, \varphi\rangle \leq \int_{\Omega} \varphi d x
$$

and conclude that $-\Delta u \leq 1$ in $\Omega$. Consequently, $u \in \mathcal{K}_{1}$ as required.

Analogously as in the previous section, we define the following functionals for the limit problem

$$
J_{1}(w):=\inf \left\{F_{1}(A): A \in \mathcal{A}_{1}^{c}(\Omega), u_{A} \leq w\right\},
$$

where $\mathcal{A}_{1}^{c}(\Omega)$ is defined in (3.7) for $s=1$ and we define $G_{1}$ to be the lower semicontinuous envolpe of $J_{1}$ in $\mathcal{K}_{1}$.

The next lemma gives the continuity of $u_{A}^{s}$ when $s \uparrow 1$.

Lemma 4.2. For every $A \in \mathcal{A}_{1}(\Omega), u_{A}^{s} \rightarrow u_{A}$ strongly in $L^{2}(\Omega)$, when $s \uparrow 1$.

Proof. Let us remind that, from Lemma $3.1, u_{A}^{s}$ is also the solution to the minimization problem

$$
I_{s}\left(u_{A}^{s}\right)=\min \left\{I_{s}(w): w \in L^{2}(\Omega)\right\}
$$

where

$$
I_{s}(w)= \begin{cases}\frac{c(n, s)}{2}[w]_{s}^{2}-\int_{\Omega} w d x & \text { if } w \in H_{0}^{s}(A), \\ \infty & \text { otherwise. }\end{cases}
$$

Notice that, by [28], we have that $\frac{c(n, s)}{2}[w]_{s}^{2} \stackrel{\Gamma}{\rightarrow} \frac{1}{2}\|\nabla w\|_{2}^{2}$. Since the $\Gamma$-convergence is stable under continuous perturbations, we have that $I_{s} \stackrel{\Gamma}{\rightarrow} I_{1}$ in $L^{2}(\Omega)$, where

$$
I_{1}(w)= \begin{cases}\frac{1}{2}\|\nabla w\|_{2}^{2}-\int_{\Omega} w d x & \text { if } w \in H_{0}^{1}(A), \\ \infty & \text { otherwise. }\end{cases}
$$

Thus, the minimizer of $I_{s}$ converges to the minimizer of $I_{1}$. That is $u_{A}^{s} \rightarrow u_{A}$ strongly in $L^{2}(\Omega)$.

Now we address the more difficult problem of understanding the limit behavior of $u_{A}^{s}$ when the domains also are varying with $s$.

This first lemma is key in understanding this limit behavior and the ideas are taken from [9]. 
Lemma 4.3. Let $0<s_{k} \uparrow 1$ and for every $k \in \mathbb{N}$ let $A_{k} \in \mathcal{A}_{s_{k}}(\Omega)$ be such that $u_{A_{k}}^{s_{k}} \rightarrow u$ strongly in $L^{2}(\Omega)$. Let $\left\{w_{k}\right\}_{k \in \mathbb{N}} \subset L^{2}(\Omega)$ be such that $w_{k} \in H_{0}^{s_{k}}\left(A_{k}\right)$ for every $k \in \mathbb{N}$ and $\sup _{k \in \mathbb{N}}\left(1-s_{k}\right)\left[w_{k}\right]_{s_{k}}^{2}<\infty$. Assume, moreover that $w_{k} \rightarrow w$ strongly in $L^{2}(\Omega)$. Then, $w \in H_{0}^{1}(\{u>0\})$.

Proof. We need to show that $w=0$ in $\mathbb{R}^{n} \backslash\{u>0\}$, i.e., $w=0$ in $\{u=0\}$.

Let us define the functional

$$
\Phi_{k}(v)= \begin{cases}\frac{c\left(n, s_{k}\right)}{2}[v]_{s_{k}}^{2} & \text { if } v \in H_{0}^{s_{k}}\left(A_{k}\right), \\ +\infty & \text { otherwise. }\end{cases}
$$

defined in $L^{2}(\Omega)$. By the compactness of $\Gamma$-convergence, there exists a subsequence still denote by $\Phi_{k}$ such that

$$
\Phi_{k} \stackrel{\Gamma}{\rightarrow} \Phi \quad \text { in } L^{2}(\Omega) .
$$

From [11, Theorem 11.10], $\Phi$ is a quadratic form in $L^{2}(\Omega)$ with domain $D(\Phi) \subset$ $L^{2}(\Omega)$.

Observe that $w \in D(\Phi)$, since

$$
\Phi(w) \leq \liminf _{k \rightarrow+\infty} \Phi_{k}\left(w_{k}\right) \leq \sup _{k \in \mathbb{N}} \frac{c\left(n, s_{k}\right)}{2}\left[w_{k}\right]_{s_{k}}^{2} \leq C \sup _{k \in \mathbb{N}}\left(1-s_{k}\right)\left[w_{k}\right]_{s_{k}}^{2}<\infty .
$$

Let $B: D(\Phi) \times D(\Phi) \rightarrow \mathbb{R}$ be the bilinear form associeted to $\Phi$, which is defined by

$$
B(v, \eta)=\frac{1}{4}(\Phi(v+\eta)-\Phi(v-\eta)) .
$$

Let us denote by $V$ the closure of $D(\Phi)$ in $L^{2}(\Omega)$ and consider the linear operator $T: D(T) \subset L^{2}(\Omega) \rightarrow L^{2}(\Omega)$ defined as $T v=f$ where

$$
D(T)=\left\{v \in D(\Phi): \exists f \in V \text { such that } B(v, \eta)=\int_{\Omega} f \eta d x, \forall \eta \in D(\Phi)\right\} .
$$

By [11, Proposition 12.17], $D(T)$ is dense in $D(\Phi)$ with respect to the norm

$$
\|v\|_{\Phi}=\left(\|v\|_{L^{2}(\Omega)}+\Phi(v)\right)^{\frac{1}{2}} .
$$

Moreover, the following relation holds

$$
\sqrt{2}\|\cdot\|_{\Phi} \geq\|\cdot\|_{H_{0}^{1}(\Omega)} .
$$

Indeed, if $z \in D(\Phi)$, as $\Phi_{k} \stackrel{\Gamma}{\rightarrow} \Phi$ in $L^{2}(\Omega)$, there exists $\left\{z_{k}\right\}_{k \in \mathbb{N}}$ such that $z_{k} \rightarrow z$ in $L^{2}(\Omega)$ and

$$
\infty>\Phi(z)=\lim _{k \rightarrow \infty} \Phi_{k}\left(z_{k}\right)= \begin{cases}\lim _{k \rightarrow \infty} \frac{c\left(n, s_{k}\right)}{2}\left[z_{k}\right]_{s_{k}}^{2} & \text { if } z_{k} \in H_{0}^{s_{k}}\left(A_{k}\right), \\ \infty & \text { otherwise. }\end{cases}
$$

Thus, $z_{k} \in H_{0}^{s_{k}}\left(A_{k}\right)$ and then

$$
\|z\|_{H_{0}^{1}(\Omega)}^{2} \leq \liminf _{k \rightarrow \infty} c\left(n, s_{k}\right)\left[z_{k}\right]_{s_{k}}^{2}=2 \lim _{k \rightarrow \infty} \Phi_{k}\left(z_{k}\right)=2 \Phi(z) \leq 2\|z\|_{\Phi}^{2} .
$$

Since (4.2) holds, $D(T)$ is dense in $D(\Phi)$ with respect to the strong topology of $H_{0}^{1}(\Omega)$. Now to achieve the proof it is enough to prove that $v=0$ in $\{u=0\}$ for all $v \in D(T)$. 
Let $v \in D(T)$ and let $f \in T v$; then $v$ is a minimum point of the functionl

$$
\Psi(\eta)=\frac{1}{2} \Phi(\eta)-\int_{\Omega} f \eta d x
$$

(see, [11, Proposition 12.12]). Let $v_{k}$ be the minimum point of functional

$$
\Psi_{k}(\eta):=\frac{1}{2} \Phi_{k}(\eta)-\int_{\Omega} f \eta d x
$$

then $v_{k}$ is the solution of the problem

$$
(-\Delta)^{s_{k}} v_{k}=f, \quad v \in H_{0}^{s_{k}}\left(A_{k}\right) .
$$

Since $\Phi_{k} \stackrel{\Gamma}{\rightarrow} \Phi$, then $\Psi_{k} \stackrel{\Gamma}{\rightarrow} \Psi$ and so we have that $v_{k} \rightarrow v$ strongly in $L^{2}(\Omega)$.

For $\varepsilon>0$ we consider $f^{\varepsilon}$ to be a bounded function with compact support such that $\left\|f^{\varepsilon}-f\right\|_{2}<\varepsilon$ and $v_{k}^{\varepsilon}$ is solution of

$$
(-\Delta)^{s_{k}} v_{k}^{\varepsilon}=f^{\varepsilon} \text { in } A_{k}, \quad v_{k}^{\varepsilon} \in H_{0}^{s_{k}}\left(A_{k}\right) .
$$

By using the linearity of the operator together with Hölder's and Poincaré's inequalities we get

$$
\begin{aligned}
\frac{c\left(n, s_{k}\right)}{2}\left[v_{k}^{\varepsilon}-v_{k}\right]_{s_{k}}^{2} & =\int_{\Omega}\left(f^{\varepsilon}-f\right)\left(v_{k}^{\varepsilon}-v_{k}\right) d x \\
& \leq\left\|f_{\varepsilon}-f\right\|_{2}\left\|v_{k}^{\varepsilon}-v_{k}\right\|_{2} .
\end{aligned}
$$

From Poincaré's inequality we obtain that

$$
\left(1-s_{k}\right)\left[v_{k}^{\varepsilon}-v_{k}\right]_{s_{k}}^{2} \leq C \varepsilon^{2},
$$

where $C$ is independent on $k$.

Then, from [3, Theorem 4], up to a subsequence, $v_{k}^{\varepsilon} \rightarrow v^{\varepsilon}$ strongly in $L^{2}(\Omega)$ and $\left\|v^{\varepsilon}-v\right\|_{H_{0}^{1}(\Omega)} \leq C \varepsilon$. At this point is enough to prove that $v^{\varepsilon}=0$ in $\{u=0\}$ for all $\varepsilon>0$.

Since $f^{\varepsilon} \leq c^{\varepsilon}:=\left\|f^{\varepsilon}\right\|_{\infty}$ and

$$
(-\Delta)^{s_{k}} v_{k}^{\varepsilon}=f^{\varepsilon} \leq c^{\varepsilon}=(-\Delta)^{s_{k}}\left(c^{\varepsilon} u_{A_{k}}^{s_{k}}\right) \text { in } A_{k}, \quad v_{k}^{\varepsilon}=c^{\varepsilon} u_{A_{k}}^{s_{k}}=0 \text { in } \mathbb{R}^{n} \backslash A_{k},
$$

the comparison principle gives that $v_{k}^{\varepsilon} \leq c^{\varepsilon} u_{A_{k}}^{s_{k}}$. Analogously, $-v_{k}^{\varepsilon} \leq c^{\varepsilon} u_{A_{k}}^{s_{k}}$.

As $k \rightarrow \infty$, we obtain that $\left|v^{\varepsilon}\right| \leq c^{\varepsilon} u$, which implies that $v^{\varepsilon}=0$ in $\{u=0\}$ for any $\varepsilon>0$ and that completes the proof.

The next lemma is the counterpart of Lemma 3.6 of the previous section. We include here the details for completeness. The main modifications with respect to the previous proof (which was analogous to that of Lemmas 3.2 and 3.3 in [9]) where carried out in the previous Lemmas of this section.

Lemma 4.4. Let $0<s_{k} \uparrow 1$ and for every $k \in \mathbb{N}$, let $A_{k} \in \mathcal{A}_{s_{k}}(\Omega), A \in \mathcal{A}_{1}(\Omega)$. Assume that $u_{A_{k}}^{s_{k}} \rightarrow u$ in $L^{2}(\Omega)$ and that $u \leq u_{A}$.

Then, if $u_{A_{k} \cup A^{\varepsilon}}^{s_{k}} \rightarrow u^{\varepsilon}$ strongly in $L^{2}(\Omega)$, where $A^{\varepsilon}:=\left\{u_{A}>\varepsilon\right\}$, it holds that $u^{\varepsilon} \leq u_{A}$.

Proof. By Lemma 3.2 with $s=1$, the inequality $u^{\varepsilon} \leq u_{A}$ will follow if we prove that $u^{\varepsilon} \in H_{0}^{1}(\Omega), u^{\varepsilon} \leq 0$ in $\mathbb{R}^{n} \backslash A$ and $-\Delta u^{\varepsilon} \leq 1$ in $\Omega$. 
Observe that by Lemma 4.1 we have that $u, u^{\varepsilon} \in H_{0}^{1}(\Omega)$. Let us define

$$
v^{\varepsilon}:=1-\frac{1}{\varepsilon} \min \left\{u_{A}, \varepsilon\right\}=\frac{1}{\varepsilon}\left(\varepsilon-u_{A}\right)^{+} .
$$

and observe that $0 \leq v^{\varepsilon} \leq 1$ and $v^{\varepsilon}=0$ in $A^{\varepsilon}$ since $0 \leq \min \left\{u_{A}, \varepsilon\right\} \leq \varepsilon$ and $\frac{1}{\varepsilon} \min \left\{u_{A}, \varepsilon\right\}=1$ in $A^{\varepsilon}$. If we define

$$
u_{k, \varepsilon}:=u_{A_{k} \cup A^{\varepsilon}}^{s_{k}}, \quad w_{k, \varepsilon}:=\min \left\{v^{\varepsilon}, u_{k, \varepsilon}\right\},
$$

it holds that $w_{k, \varepsilon} \geq 0$ since the comparison principle gives $u_{k, \varepsilon} \geq 0$, and also $v^{\varepsilon} \geq 0$.

Since $v^{\varepsilon}=0$ in $A^{\varepsilon}$, it follows that $w_{k, \varepsilon}=0$ in $A^{\varepsilon}$. Moreover, since $u_{k, \varepsilon}=0$ in $\mathbb{R}^{n} \backslash\left(A_{k} \cup A^{\varepsilon}\right)$, it holds that $w_{k, \varepsilon}=0$ in $\mathbb{R}^{n} \backslash\left(A_{k} \cup A^{\varepsilon}\right)$, and consequently, $w_{k, \varepsilon} \in H_{0}^{s_{k}}\left(A_{k}\right)$.

Notice that $w_{k, \varepsilon} \rightarrow w_{\varepsilon}:=\min \left\{v^{\varepsilon}, u^{\varepsilon}\right\}$ strongly in $L^{2}(\Omega)$, and then, applying Lemma 4.3 , we get $w_{\varepsilon} \in H_{0}^{1}(\{u>0\})$, from where $w_{\varepsilon}=0$ in $\{u=0\}$. The relation $0 \leq u \leq u_{A}$ implies the inclusion $\left\{u_{A}=0\right\} \subset\{u=0\}$, from where $w_{\varepsilon} \in H_{0}^{1}\left(\left\{u_{A}>0\right\}\right)$. Moreover, since $\left\{u_{A}>0\right\} \subset A$, we have that $w_{\varepsilon}=0$ in $\mathbb{R}^{n} \backslash A$. Now, being $v^{\varepsilon}=1$ in $\mathbb{R}^{n} \backslash A$, we get $u^{\varepsilon}=0$ in $\mathbb{R}^{n} \backslash A$, and in particular, $u^{\varepsilon} \leq 0$ in $\mathbb{R}^{n} \backslash A$.

Finally, it remains to see that $-\Delta u^{\varepsilon} \leq 1$ in $\Omega$. Observe that $u_{k, \varepsilon} \in \mathcal{K}_{s_{k}}$ and $u_{k, \varepsilon} \rightarrow u^{\varepsilon}$ strongly in $L^{2}(\Omega)$. Then $u^{\varepsilon} \in \overline{\mathcal{K}}_{1}$ by Lemma 4 .1. Thus $-\Delta u^{\varepsilon} \leq 1$ in $\Omega$ and the proof is complete.

With the help of these lemmas, we are now in position to prove then main tool needed in the proof of Theorem 2.10.

Proposition 4.5. Let $0<s_{k} \uparrow 1, A_{k} \in \mathcal{A}_{s_{k}}^{c}(\Omega)$ be such that $u_{A_{k}}^{s_{k}} \rightarrow u$ strongly in $L^{2}(\Omega)$. Then, there exist $\tilde{A}_{k} \in \mathcal{A}_{s_{k}}(\Omega)$ such that $A_{k} \subset \tilde{A}_{k}$ and $\tilde{A}_{k} \gamma$-converges to $A:=\{u>0\}$.

Proof. Since $u_{A_{k}}^{s_{k}} \in \mathcal{K}_{s_{k}}$ and $u_{A_{k}}^{s_{k}} \rightarrow u$, by Lemma 4.1, $u \in \mathcal{K}_{1}$. Then, by Lemma $3.2, u \leq u_{A}$.

As in the previous proof, consider $A^{\varepsilon}:=\left\{u_{A}>\varepsilon\right\}$ and observe that

$$
u_{A^{\varepsilon}}^{s_{k}} \leq u_{A_{k} \cup A^{\varepsilon}}^{s_{k}}
$$

Since $u_{A_{k} \cup A^{\varepsilon}}^{s_{k}} \in \mathcal{K}_{s_{k}}$, by Lemma 4.1 , there exists $u^{\varepsilon} \in H_{0}^{1}(\Omega)$ such that $u_{A_{k} \cup A^{\varepsilon}}^{s_{k}} \rightarrow$ $u^{\varepsilon}$ strongly in $L^{2}(\Omega)$, up to a subsequence.

Also, by Lemma $4.2, u_{A^{\varepsilon}}^{s_{k}} \rightarrow u_{A^{\varepsilon}}$ strongly in $L^{2}(\Omega)$. Then, we can pass to the limit as $k \rightarrow \infty$ in the previous inequality to conclude that

$$
u_{A^{\varepsilon}} \leq u^{\varepsilon}
$$

It can be easily checked that $u_{A^{\varepsilon}}=\left(u_{A}-\varepsilon\right)_{+}$. Moreover, from Lemma 4.4,

$$
\left(u_{A}-\varepsilon\right)_{+} \leq u^{\varepsilon} \leq u_{A}
$$

Thus, there exists a sequence $0<\varepsilon_{k} \downarrow 0$ such that

$$
u_{A_{k} \cup A^{\varepsilon_{k}}}^{s_{k}} \rightarrow u_{A} \text { strongly in } L^{2}(\Omega) .
$$

That is, $A_{k} \cup A^{\varepsilon_{k}}=: \tilde{A}_{k} \gamma$-converges to $A$.

Now we are ready to prove the main result. 
Proof of Theorem 2.10. By Theorem 2.7, there exists $A_{k} \in \mathcal{A}_{s_{k}}^{c}(\Omega)$ such that

$$
F_{s_{k}}\left(A_{k}\right)=\min \left\{F_{s_{k}}(A): A \in \mathcal{A}_{s_{k}}^{c}(\Omega)\right\} .
$$

Then, if $A \in \mathcal{A}_{1}^{c}(\Omega)$, by condition $\left(H_{1}\right)$ we know that

$$
\limsup _{k \rightarrow \infty} F_{s_{k}}\left(A_{k}\right) \leq \lim _{k \rightarrow \infty} F_{s_{k}}(A)=F_{1}(A),
$$

from where it follows that

$$
\limsup _{k \rightarrow \infty} \min \left\{F_{s_{k}}(A): A \in \mathcal{A}_{s_{k}}^{c}(\Omega)\right\} \leq \min \left\{F_{1}(A): A \in \mathcal{A}_{1}^{c}(\Omega)\right\} .
$$

Let us see the reverse inequality. By simplicity, let us denote $u_{k}:=u_{A_{k}}^{s_{k}} \in \mathcal{K}_{s_{k}}$.

By Lemma 4.1 , there is $u \in H_{0}^{1}(\Omega)$ such that, up to a subsequence, $u_{k} \rightarrow u$ strongly in $L^{2}(\Omega)$.

Moreover, by Proposition 4.5, there exists $\tilde{A}_{k} \in \mathcal{A}_{s_{k}}(\Omega)$ such that $A_{k} \subset \tilde{A}_{k}$ and $\tilde{A}_{k} \gamma$-converges to $A:=\{u>0\}$. Since $u_{k} \rightarrow u$ in $L^{2}(\Omega),|A| \leq c$.

Finally, from condition $\left(H_{2}\right)$ and $\left(H_{2}^{s}\right)$ we conclude that

$$
F_{1}(A) \leq \liminf _{k \rightarrow \infty} F_{s_{k}}\left(\tilde{A}_{k}\right) \leq \liminf _{k \rightarrow \infty} F_{s_{k}}\left(A_{k}\right),
$$

from where it follows that

$$
\min \left\{F_{1}(A): A \in \mathcal{A}_{1}^{c}(\Omega)\right\} \leq \liminf _{k \rightarrow \infty} \min \left\{F_{s_{k}}(A): A \in \mathcal{A}_{s_{k}}^{c}(\Omega)\right\} .
$$

Putting together (4.3) and (4.4) the result follows.

\section{ACKNOWLEDGEMENTS}

This paper was partially supported by grants UBACyT 20020130100283BA, CONICET PIP 11220150100032CO and ANPCyT PICT 2012-0153.

J. Fernández Bonder and Ariel M. Salort are members of CONICET and A. Ritorto is a doctoral fellow of CONICET.

\section{REFERENCES}

1. Vedat Akgiray and G. Geoffrey Booth, The siable-law model of stock returns, Journal of Business \& Economic Statistics 6 (1988), no. 1, 51-57.

2. Grégoire Allaire, Shape optimization by the homogenization method, Applied Mathematical Sciences, vol. 146, Springer-Verlag, New York, 2002. MR 1859696 (2002h:49001)

3. Jean Bourgain, Haim Brezis, and Petru Mironescu, Another look at sobolev spaces, in Optimal Control and Partial Differential Equations, 2001, pp. 439-455.

4. Lorenzo Brasco and Enea Parini, The second eigenvalue of the fractional p-Laplacian, Adv. Calc. Var. 9 (2016), no. 4, 323-355. MR 3552458

5. Lorenzo Brasco, Enea Parini, and Marco Squassina, Stability of variational eigenvalues for the fractional p-Laplacian, Discrete Contin. Dyn. Syst. 36 (2016), no. 4, 1813-1845. MR 3411543

6. Haim Brezis, Functional analysis, Sobolev spaces and partial differential equations, Universitext, Springer, New York, 2011. MR 2759829

7. Dorin Bucur and Giuseppe Buttazzo, Variational methods in shape optimization problems, Progress in Nonlinear Differential Equations and their Applications, 65, Birkhäuser Boston, Inc., Boston, MA, 2005. MR 2150214

8. Almut Burchard, Rustum Choksi, and Ihsan Topaloglu, Nonlocal shape optimization via interactions of attractive and repulsive potentials, arXiv preprint arXiv:1512.07282 (2015).

9. Giuseppe Buttazzo and Gianni Dal Maso, An existence result for a class of shape optimization problems, Arch. Rational Mech. Anal. 122 (1993), no. 2, 183-195. MR 1217590 
10. Peter Constantin, Euler equations, Navier-Stokes equations and turbulence, Mathematical foundation of turbulent viscous flows, Lecture Notes in Math., vol. 1871, Springer, Berlin, 2006, pp. 1-43. MR 2196360

11. Gianni Dal Maso, An introduction to $\Gamma$-convergence, Progress in Nonlinear Differential Equations and their Applications, 8, Birkhäuser Boston, Inc., Boston, MA, 1993. MR 1201152

12. Anne-Laure Dalibard and David Gérard-Varet, On shape optimization problems involving the fractional Laplacian, ESAIM Control Optim. Calc. Var. 19 (2013), no. 4, 976-1013. MR 3182677

13. Eleonora Di Nezza, Giampiero Palatucci, and Enrico Valdinoci, Hitchhiker's guide to the fractional Sobolev spaces, Bull. Sci. Math. 136 (2012), no. 5, 521-573. MR 2944369

14. Qiang Du, Max Gunzburger, R. B. Lehoucq, and Kun Zhou, Analysis and approximation of nonlocal diffusion problems with volume constraints, SIAM Rev. 54 (2012), no. 4, 667-696. MR 3023366

15. A. Cemal Eringen, Nonlocal continuum field theories, Springer-Verlag, New York, 2002. MR 1918950

16. J. Fernandez Bonder and J. Spedaletti, Some nonlocal optimal design problems, ArXiv e-prints (2016).

17. Giambattista Giacomin and Joel L. Lebowitz, Phase segregation dynamics in particle systems with long range interactions. I. Macroscopic limits, J. Statist. Phys. 87 (1997), no. 1-2, 37-61. MR 1453735

18. Guy Gilboa and Stanley Osher, Nonlocal operators with applications to image processing, Multiscale Model. Simul. 7 (2008), no. 3, 1005-1028. MR 2480109

19. Antoine Henrot, Extremum problems for eigenvalues of elliptic operators, Frontiers in Mathematics, Birkhäuser Verlag, Basel, 2006. MR 2251558

20. Nicolas et al. Humphries, Environmental context explains lévy and brownian movement patterns of marine predators, Nature 465 (2010), 1066-1069.

21. Hans Knüpfer and Cyrill B. Muratov, On an isoperimetric problem with a competing nonlocal term I: The planar case, Comm. Pure Appl. Math. 66 (2013), no. 7, 1129-1162. MR 3055587

22. _ On an isoperimetric problem with a competing nonlocal term II: The general case, Comm. Pure Appl. Math. 67 (2014), no. 12, 1974-1994. MR 3272365

23. Nikolai Laskin, Fractional quantum mechanics and Lévy path integrals, Phys. Lett. A 268 (2000), no. 4-6, 298-305. MR 1755089

24. Sergei Levendorski, Pricing of the american put under lévy processes, International Journal of Theoretical and Applied Finance 7 (2004), no. 03, 303-335.

25. A. Massaccesi and E. Valdinoci, Is a nonlocal diffusion strategy convenient for biological populations in competition?, ArXiv e-prints (2015).

26. Ralf Metzler and Joseph Klafter, The random walk's guide to anomalous diffusion: a fractional dynamics approach, Phys. Rep. 339 (2000), no. 1, 77. MR 1809268

27. Olivier Pironneau, Optimal shape design for elliptic systems, Springer Series in Computational Physics, Springer-Verlag, New York, 1984. MR 725856

28. Augusto C. Ponce, A new approach to Sobolev spaces and connections to $\Gamma$-convergence, Calc. Var. Partial Differential Equations 19 (2004), no. 3, 229-255. MR 2033060

29. Chong Qiu, Yisheng Huang, and Yuying Zhou, Optimization problems involving the fractional Laplacian, Electron. J. Differential Equations (2016), Paper No. 98, 15. MR 3489970

30. A. M. Reynolds and C. J. Rhodes, The lvy flight paradigm: random search patterns and mechanisms, Ecology 90 (2009), no. 4, 877-887.

31. Wim Schoutens, Lévy processes in finance: Pricing financial derivatives, Willey Series in Probability and Statistics, Willey, New York, 2003.

32. Shaoguang Shi and Jie Xiao, On fractional capacities relative to bounded open Lipschitz sets, Potential Anal. 45 (2016), no. 2, 261-298. MR 3518675

33. Y. Sire, J. L. Vazquez, and B. Volzone, Symmetrization for fractional elliptic and parabolic equations and an isoperimetric application, ArXiv e-prints (2015).

34. Jan Sokołowski and Jean-Paul Zolésio, Introduction to shape optimization, Springer Series in Computational Mathematics, vol. 16, Springer-Verlag, Berlin, 1992, Shape sensitivity analysis. MR 1215733

35. Elias M. Stein, Singular integrals and differentiability properties of functions, Princeton Mathematical Series, No. 30, Princeton University Press, Princeton, N.J., 1970. MR 0290095 
36. Kun Zhou and Qiang Du, Mathematical and numerical analysis of linear peridynamic models with nonlocal boundary conditions, SIAM J. Numer. Anal. 48 (2010), no. 5, 1759-1780. MR 2733097

Departamento de Matemática, FCEn - Universidad de Buenos Aires and imas COnicet, Buenos Aires, Argentina

E-mail address, J. Fernández Bonder: jfbonder@dm.uba.ar

$U R L$, J. Fernández Bonder: http://mate.dm.uba.ar/ jfbonder

E-mail address, A. Ritorto: aritorto@dm.uba.ar

E-mail address, A.M. Salort: asalort@dm.uba.ar

$U R L$, A.M. Salort: http://mate.dm.uba.ar/ asalort 